\title{
Un système de vigie rehaussée de santé publique pour un rassemblement de masse
}

\author{
C Huot ${ }^{1 *}$, A Paradis², K Hammond-Collins ${ }^{3}$, MA Bélair ${ }^{4}$, J Villeneuve ${ }^{5}$, N Brousseau', \\ I Goupil-Sormany ${ }^{1,2}$, J Riffon ${ }^{1}$
}

\section{Résumé}

Contexte : Du 7 au 9 juin 2018, un Sommet du G7 a eu lieu dans la province de Québec. Ce rassemblement de masse de nature politique à portée internationale présentait un certain nombre de risques potentiels pour la santé publique.

Objectif : Évaluer trois stratégies de vigie supplémentaires afin de détecter les menaces pouvant survenir lors d'un rassemblement de masse.

Intervention : En plus des activités usuelles de vigie de santé publique, un partenariat a été créé et trois stratégies de vigie supplémentaires ont été mises en œuvre trois jours avant, pendant et six jours après l'événement du G7 : I'analyse des données des raisons de consultation et des diagnostics médicaux de 11 urgences de la région à l'aide du logiciel « Early Aberration Reporting System »; l'envoi quotidien d'un questionnaire en ligne à des partenaires clés; et l'analyse des raisons d'appel à Info-Santé, un service gouvernemental de consultation téléphonique pour des problèmes de santé et sociaux.

Résultats : Les données des urgences ont généré 78 alertes à partir des raisons de consultation et 39 alertes pour les diagnostics médicaux. Parmi ces 117 alertes, deux ont fait l'objet d'une enquête (une dans la catégorie respiratoire et l'autre dans la catégorie neurologique/ musculaire) et aucune intervention supplémentaire n'a été nécessaire. À quelques exceptions près, tous les partenaires ont rempli le sondage en ligne chaque jour et aucun signal n'a été généré. En comparaison avec les données historiques, aucune augmentation ou différence dans les données des appels à Info-Santé n'a été détectée pendant la période de vigie.

Conclusion : Les trois stratégies de vigie supplémentaires élaborées pour détecter des événements de santé publique au cours du Sommet du G7 au Québec ont permis de recueillir des données pertinentes et en temps opportun aux fins d'analyse. Une collaboration étroite et une bonne participation des différents partenaires étaient essentielles pour ce projet.

Cependant, étant donné qu'aucun événement de santé publique n'a eu lieu, il n'a pas été possible de déterminer si le système de vigie rehaussée disposait d'assez de réactivité et de sensibilité pour une détection et une intervention rapides.
Cette oeuvre est mise à la disposition selon les termes de la licence internationale Creative Commons Attribution 4.0

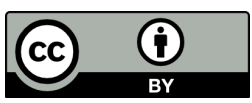

Affiliations

${ }^{1}$ Direction de santé publique du Centre intégré universitaire de santé et de services sociaux de la Capitale-Nationale, Québec, QC

${ }^{2}$ Département de médecine sociale et préventive, Université Laval, Québec, QC

${ }^{3}$ Centre for Food-borne, Environmental and Zoonotic Infecious Diseases, Public Health Agency of Canada, Ottawa, ON

${ }^{4}$ Centre de mesures et d'interventions d'urgence, Agence de la santé publique du Canada, Whitehorse, YK

${ }^{5}$ Institut national de santé publique du Québec, Québec, QC

*Correspondance:

caroline.huot@inspq.qc.ca

Suggested citation: Huot C, Paradis A, Hammond-Collins K, Bélair MA, Villeneuve J, Brousseau N,

Goupil-Sormany I, Riffon J. Un système de vigie rehaussée de santé publique pour un rassemblement de masse. Relevé des maladies transmissibles au Canada 2019;45(7/8):233-46. https://doi.org/10.14745/ccdr.v45i78a05f

Keywords: rassemblement de masse, surveillance, surveillance syndromique, santé publique, épidémiologie

\section{Introduction}

Du 7 au 9 juin 2018, le premier ministre du Canada a tenu un Sommet du G7 à La Malbaie et à Québec, Québec (1). Sept personnes jouissant d'une protection internationale et leurs délégations (environ 3200 personnes), 3200 journalistes, des milliers de manifestants, 1000 à 2000 policiers, 1000 membres des Forces armées canadiennes et environ 12000 personnes de pays non-membres du G7, invités dans le cadre du Programme « Outreach », y étaient attendus. 
En préparation à cet événement, les experts en gestion des urgences ont été consultés et la littérature pertinente (2-9), y compris les leçons tirées du Sommet des Amériques en 2001 (10), a été examinée. Un certain nombre d'initiatives de vigie entreprises par le passé au cours d'événements sportifs $(3,11-$ 19), religieux $(5,20,21)$ et artistiques $(22,23)$ ont déjà été décrites. Cependant, moins d'information détaillée était disponible à propos de la surveillance des événements politiques, en particulier ceux présentant un risque de manifestations violentes ou d'actes terroristes $(24,25)$.

Avant l'événement, certaines menaces potentielles à la santé publique ont été identifiées et priorisées aux fins de vigie: propagation rapide de certaines maladies infectieuses; violence liée aux manifestations et déploiement d'agents de contrôle de foule; potentiel de menace terroriste chimique, biologique, radiologique, nucléaire ou explosive (CBRNE); et présence de colis suspects (26). Bien que la probabilité de survenue de ces menaces ait été considérée comme faible (11-16,27), un système de vigie rehaussée pour rapidement détecter les menaces potentielles à la santé publique (28-31) ainsi qu'un plan d'intervention pour chacune de ces menaces étaient nécessaires.

La vigie usuelle de santé publique au Québec repose sur la déclaration des maladies à déclaration obligatoire par les médecins et les laboratoires et sur les signalements passifs des menaces perçues ou réelles à la santé publique par divers partenaires, y compris les médecins, les autorités gouvernementales et les municipalités locales (32). Cependant, ces activités de vigie n'avaient pas une sensibilité et une réactivité optimales pour rapidement détecter et réagir aux menaces priorisées par la santé publique lors du G7 (33). Pour y remédier, la Direction de santé publique (DSPublique) du Centre intégré universitaire de santé et de services sociaux (CIUSSS) de la Capitale-Nationale a développé un système de vigie rehaussée pour la période du 4 au 15 juin 2018 (trois jours avant, trois jours pendant et six jours après l'événement). L'objectif du système de vigie rehaussée était de détecter précocement toute menace de santé publique tout en demeurant acceptable pour tous les partenaires participants.

Le but du présent article est de décrire l'élaboration et les résultats de la mise en œuvre de ces trois stratégies qui faisaient partie du système de vigie rehaussée au cours du Sommet du G7 2018 ainsi que considérer les implications pour la vigie de santé publique au cours de prochains événements de masse.

\section{Établir des partenariats}

Environ six mois avant l'événement, une équipe de projet de la DSPublique a commencé à mettre en place le système de vigie rehaussée. Toutes les urgences de la région avaient déjà un système informatisé de saisie des raisons de consultations (par les infirmières du triage à l'arrivée des patients) et des diagnostics médicaux (donnés par un médecin lorsque le patient quitte l'urgence). Toutes ces données ne sont généralement pas accessibles à la DSPublique et elles n'ont jamais été utilisées pour la vigie de santé publique. Les urgences de six centres hospitaliers universitaires et de cinq centres hospitaliers régionaux dans le périmètre de l'événement et les alentours ont été invitées à collaborer dans le cadre de cet événement. Pour assurer l'accès à ces données, de nouveaux partenaires d'autres directions du CIUSSS ont collaboré avec l'équipe de projet de la DSPublique.

Afin de recevoir des rapports quotidiens des partenaires clés, cinq organisations susceptibles de détecter rapidement les menaces ciblées ont été approchées : le Centre antipoison du Québec, les services ambulanciers de la région, le Laboratoire de santé publique du Québec et le Bureau du coroner. Info-Santé (un service de consultation téléphonique pour des problèmes de santé et sociaux) est un partenaire clé qui a soumis des rapports quotidiens et fourni des données sur les raisons d'appel. Trois cliniques temporaires ont été mises en place pour l'événement et elles ont également rempli des rapports quotidiens.

En plus des partenaires qui ont directement contribué au système de vigie rehaussée, les médecins de la région ont été informés de la progression du projet, étape par étape, par le biais de la structure de sécurité civile, qui a été mise en alerte pour l'événement. L'Agence de la santé publique du Canada a apporté son soutien, en déployant deux épidémiologistes de terrain pour la planification, l'analyse des données et la production de rapports. Toutes les activités de vigie rehaussée ont été coordonnées afin de rendre plus rapides l'analyse, la diffusion des résultats, la prise de décisions et les interventions appropriées. Un rapport quotidien résumant les résultats a été préparé et distribué aux partenaires et décideurs. Deux versions (courte et détaillée), adaptées aux publics cibles, étaient disponibles.

\section{Activités de vigie rehaussée}

Afin d'améliorer la sensibilité et la rapidité du système de vigie tout en s'assurant qu'il reste acceptable pour tous les partenaires, les trois activités de vigie suivantes ont été ajoutées au système de déclaration des maladies à déclaration obligatoire et au signalement passif des menaces à la santé pour la période du 4 au 15 juin 2018 :

- Vigie des raisons de consultation et des diagnostics médicaux dans les urgences

- Rapports quotidiens des partenaires clés

- Vigie des tendances des raisons d'appel à Info-Santé 


\section{Vigie des raisons de consultation et des diagnostics médicaux dans les urgences}

Pour les données électroniques des urgences, les raisons de consultation ont été enregistrées par les infirmières au triage à l'arrivée d'un patient et les diagnostics médicaux à la sortie de l'urgence. Tous les patients des hôpitaux universitaires ont été identifiés grâce à un numéro d'identifiant unique. Ces données étaient disponibles le jour suivant la visite. Les données de toutes les visites dans les urgences aux alentours du Sommet du G7 ont été intégrées dans le système de vigie rehaussée. Cette approche était acceptable pour les partenaires cliniques car ces données étaient déjà disponibles pour d'autres fins. Cependant, cela a créé une légère augmentation de la charge de travail puisque des rappels ont été envoyés en cas de retards de saisie.

Les diagnostics médicaux ont été identifiés selon la Classification internationale des maladies, 10 e révision (CIM-10) en vertu du cadre normatif pour le système informatisé du Québec (34). Les raisons de consultation et les diagnostics médicaux sélectionnés ont été classés et analysés par catégorie (voir les annexes 1 et 2). Quelques diagnostics médicaux ont aussi été analysés individuellement; soit parce qu'ils représentaient une maladie à déclaration obligatoire ou qu'il s'agissait d'un diagnostic pour lequel une intervention de santé publique pourrait être indiquée pour un cas unique, comme la rougeole (voir dernière colonne de l'Annexe 2). Deux médecins, un spécialisé dans les urgences CBRNE et l'autre en toxicologie clinique, ont validé ces choix.

Le nombre de cas dans chaque catégorie a été analysé à l'aide du logiciel «Early Aberration Reporting System » des Centers for Disease Control and Prevention (CDC) (35), qui détecte les alertes ou aberrations dans le nombre de cas par rapport aux données historiques récentes (dix jours). Toutes les alertes générées ont été comparées aux données historiques pour la période entre avril et juillet 2013-2017. Une analyse quotidienne des données du jour précédent a été réalisée, par urgence et pour toutes les urgences combinées. Le groupe d'âge, la municipalité des cas et les détails des raisons de consultation et des diagnostics médicaux ont été utilisés lorsque disponibles. Une évaluation a été réalisée et une décision a été prise en équipe quant à la nécessité d'enquêter davantage sur chaque alerte générée.

\section{Rapports quotidiens des partenaires clés}

Les partenaires clés comprenaient le Centre antipoison du Québec, les services ambulanciers de la région, le Laboratoire de santé publique du Québec, le Bureau du coroner, Info-Santé et trois cliniques temporaires. Un court questionnaire en ligne de trois questions (plateforme Voxco Inc.) (36) sur les menaces de maladies infectieuses ou de santé environnementale a été élaboré et soumis quotidiennement à ces partenaires (avant, pendant et après l'événement). Les questionnaires portaient sur les évènements survenus le jour précédent et étaient remplis avant $10 \mathrm{~h}$ chaque jour.

\section{Vigie des tendances des raisons d'appel à Info-Santé}

Les données électroniques sur les raisons d'appel à Info-Santé étaient déjà accessibles pour la DSPublique mais n'avaient jamais été utilisées auparavant à des fins de vigie. Les raisons d'appel les plus pertinentes ont été sélectionnées en fonction des menaces priorisées (tableau 1). Ces raisons ont été regroupées en catégories. Dans chaque catégorie, le nombre et le pourcentage des appels ont été analysés quotidiennement pour détecter toute augmentation ou modification en comparaison avec les données historiques.

\section{Tableau 1 : Raisons d'appel à Info-Santé, par catégorie}

\begin{tabular}{|c|c|}
\hline Catégorie & Raison d'appel \\
\hline Cardiovasculaire & $\begin{array}{l}\text { Manifestations ou symptômes du système } \\
\text { cardiovasculaire }\end{array}$ \\
\hline \multirow{4}{*}{$\begin{array}{l}\text { CBRNE/physique/ } \\
\text { environnemental }\end{array}$} & Chaleur extrème \\
\hline & Empoisonnements \\
\hline & Événements d'envergure \\
\hline & Santé environnementale \\
\hline Cutané/lymphatique & Manifestations ou symptômes peau et tissus \\
\hline \multirow[t]{2}{*}{ Extrême } & Influenza d'origine aviaire (grippe aviaire) \\
\hline & Virus Ébola \\
\hline Gastro-intestinal & $\begin{array}{l}\text { Manifestations ou symptômes du système } \\
\text { gastro-intestinal }\end{array}$ \\
\hline \multirow[t]{2}{*}{ Infectieux } & Maladies infectieuses et parasitaires \\
\hline & Thermorégulation \\
\hline $\begin{array}{l}\text { Neurologique/ } \\
\text { musculaire }\end{array}$ & $\begin{array}{l}\text { Manifestations ou symptômes du système } \\
\text { nerveux }\end{array}$ \\
\hline $\begin{array}{l}\text { Ophtalmologique/oto- } \\
\text { rhino-laryngologique }\end{array}$ & $\begin{array}{l}\text { Manifestations ou symptômes du système } \\
\text { ophtalmique }\end{array}$ \\
\hline \multirow[t]{2}{*}{ Respiratoire } & Syndrome d'allure grippale \\
\hline & Problèmes respiratoires excluant la grippe \\
\hline
\end{tabular}

\section{Résultats}

\section{Raisons de consultation et diagnostics médicaux des urgences}

Au cours de la période de vigie, les données étaient disponibles pour les raisons de consultation et les diagnostics médicaux. Aucune donnée sur les raisons de consultation n'était manquante. Globalement, $27 \%$ des données sur les diagnostics médicaux étaient manquantes pendant toute la période de vigie, qui s'étendait du 4 au 15 juin. Cependant, lors du Sommet du G7 (du 7 au 9 juin), seulement $23 \%$ des données sur les diagnostics médicaux n'étaient pas disponibles. Ces données manquantes 
étaient causées par le départ de patients des urgences avant qu'un diagnostic ne soit posé et par les retards de saisie des diagnostics dans le système informatisé.

Les données des urgences ont produit 78 alertes pour les catégories des raisons de consultation et ont généré 39 alertes dans les diverses catégories de diagnostics médicaux. De ces 117 alertes, deux ont fait l'objet d'une enquête (une pour la catégorie respiratoire et une pour la catégorie neurologique/ musculaire). Aucune intervention supplémentaire n'a été requise. Le tableau 2 résume le nombre d'alertes par catégorie.

Tableau 2 : Nombre d'alertes par catégorie de raisons de consultation et de diagnostics médicaux, 11 urgences de la région de La Malbaie/Québec, 4 au 15 juin 2018

\begin{tabular}{|c|c|c|}
\hline \multirow[b]{2}{*}{ Catégorie } & \multicolumn{2}{|c|}{ Nombre d'alertes } \\
\hline & $\begin{array}{l}\text { Raison de } \\
\text { consultation }\end{array}$ & $\begin{array}{l}\text { Diagnostics } \\
\text { médicaux }\end{array}$ \\
\hline Cardiovasculaire & 5 & 3 \\
\hline CBRNE/physique/environnemental & 6 & 4 \\
\hline Cutané/lymphatique & 6 & 4 \\
\hline Fièvre & 9 & $\mathrm{~S} / \mathrm{O}$ \\
\hline Gastro-intestinal & 9 & 0 \\
\hline Hémorragique & 8 & 6 \\
\hline Infectieux & $\mathrm{S} / \mathrm{O}$ & 5 \\
\hline Intoxication aux opioïdes & $\mathrm{S} / \mathrm{O}$ & 0 \\
\hline Neurologique/musculaire & 8 & 8 \\
\hline $\begin{array}{l}\text { Ophtalmique/oto-rhino- } \\
\text { laryngologique }\end{array}$ & 9 & 4 \\
\hline Respiratoire & 5 & 2 \\
\hline Systémique/déshydratation & 13 & 3 \\
\hline TOTAL & 78 & 39 \\
\hline
\end{tabular}

Abréviations : CBRNE, chimique, biologique, radiologique, nucléaire ou explosif; S/O, Sans objet

\section{Rapports quotidiens des partenaires clés et données d'Info-Santé}

À quelques exceptions près, tous les partenaires ont rempli le sondage chaque jour et peu de rappels ont été nécessaires. Une des trois cliniques temporaires a signalé deux cas de gastroentérite. Les services ambulanciers ont signalé un cas d'intoxication aux opioïdes. Sachant que ces nombres n'ont pas dépassé la fréquence attendue et qu'il n'y avait aucune menace à la santé associée, aucun de ces signalements n'a fait l'objet d'une enquête.

Concernant les raisons d'appel à Info-Santé, aucune augmentation ou différence n'a été détectée pendant la période de vigie en comparaison avec les données historiques.

\section{Discussion}

Un système de vigie rehaussée à trois volets, élaboré pour détecter les événements de santé publique au cours du Sommet du G7 2018 au Québec, a réussi à recueillir des données en temps opportun aux fins d'analyse. La plupart des alertes ont été générées à partir de l'analyse des données des urgences. L'utilisation des données historiques a permis de limiter le nombre d'alertes pour lesquelles une enquête a été nécessaire. L'utilisation des données des urgences semble avoir conféré une sensibilité élevée mais une faible spécificité au système.

Une force de ce système de vigie rehaussée était la collaboration étroite avec divers partenaires. Cette collaboration était essentielle à l'élaboration de ce système. Les trois stratégies de vigie supplémentaires ont fait l'objet d'un niveau de participation élevé de la part des partenaires. Ceci est probablement attribuable au fait qu'ils comprenaient l'importance d'une vigie rehaussée et que peu de temps supplémentaire était requis pour y participer. Ainsi, la plupart des données demandées aux partenaires ont été reçues en temps opportun.

Ces travaux ont confirmé la faisabilité de la mise en œuvre de ce système de vigie rehaussée et pourront soutenir la préparation de santé publique pour de futurs rassemblements de masse. Cependant, puisqu'aucun événement de santé publique n'a eu lieu, il n'a pas été possible de déterminer si le système de vigie rehaussée disposait d'assez de réactivité et de sensibilité pour une détection et une intervention suffisamment rapides

\section{Conclusion}

Trois stratégies de vigie supplémentaires élaborées pour détecter des événements de santé publique au cours du Sommet du G7 au Québec ont permis de recueillir des données pertinentes et en temps opportun pour l'analyse. Cependant, la réactivité et la sensibilité de ces stratégies devront être réévaluées si elles sont utilisées lors d'un futur rassemblement de masse.

\section{Authors' statement}

H. C. - Conceptualisation, méthodologie, intervention, analyse et interprétation des données, rédaction de l'a

A. P. - Conceptualisation, méthodologie, intervention, analyse et interprétation des données, révision de l'article

K. H. C. - Intervention, analyse et interprétation des données, révision de l'article

M. A. B. - Analyse et interprétation des données, révision de l'article

J. V. - Conceptualisation, méthodologie, révision de l'article

N. B. - Intervention, analyse et interprétation des données, révision de l'article

I. G. S. -Conceptualisation, ressources, révision de l'article J. R. - Intervention, analyse et interprétation des données, révision de l'article 


\section{Conflit of d'intérêts}

Aucun.

\section{Remerciements}

Les auteurs souhaiteraient souligner le soutien des collègues suivants : F. W. Tremblay, M. Mercier, F. Desbiens, N. Beaulieu, I. Beaulieu, C. Thibault, A. Paradis, N. Allain-Boulé, M. Beauregard, A. Maurice, N. Thériault, J. Lizotte, S. Gaudreault, J. Larocque, S. Blais, D. Martineau, V. Tremblay, R. Dion, È. Pilon, et M. St-Onge.

\section{Financement}

Ces travaux ont été soutenus par le Centre intégré universitaire de santé et de services sociaux de la Capitale-Nationale et I'Agence de la santé publique du Canada.

\section{Références}

1. Présidence canadienne du G7 de 2018. Présidence canadienne du G7 de 2018. 2018. https://international. gc.ca/world-monde/international_relations-relations_ internationales/g7/index.aspx?lang=fra\#a3

2. Kaiser R, Coulombier D. Epidemic intelligence during mass gatherings. Euro Surveill 2006 Dec;11(12):E061221.3. DOI PubMed

3. Elliot AJ, Hughes HE, Hughes TC, Locker TE, Shannon T, Heyworth J, Wapling A, Catchpole M, Ibbotson S, McCloskey $B$, Smith GE. Establishing an emergency department syndromic surveillance system to support the London 2012 Olympic and Paralympic Games. Emerg Med J 2012 Dec;29(12):954-60. DOI PubMed

4. Abubakar I, Gautret P, Brunette GW, Blumberg L, Johnson D, Poumerol G, Memish ZA, Barbeschi M, Khan AS. Global perspectives for prevention of infectious diseases associated with mass gatherings. Lancet Infect Dis 2012 Jan;12(1):6674. DOI PubMed

5. Memish ZA, Zumla A, Alhakeem RF, Assiri A, Turkestani A, Al Harby KD, Alyemni M, Dhafar K, Gautret P, Barbeschi M, McCloskey B, Heymann D, Al Rabeeah AA, Al-Tawfiq JA. Hajj: infectious disease surveillance and control. Lancet 2014 Jun;383(9934):2073-82. DOI PubMed

6. Sun X, Keim M, He Y, Mahany M, Yuan Z. Reducing the risk of public health emergencies for the world's largest mass gathering: 2010 World Exposition, Shanghai China. Disaster Health 2013 Jan;1(1):21-9. DOI PubMed

7. Menezes RG, Hussain SA, Rameez MA, Kharoshah MA, Madadin M, Anwar N, Senthilkumaran S. Chemical crowd control agents. Med Leg J 2016 Mar;84(1):22-5. DOI PubMed

8. Heggie TW. Traveling to Canada for the Vancouver 2010 Winter Olympic and Paralympic Games. Travel Med Infect Dis 2009 Jul;7(4):207-11. DOl PubMed
9. Ortiz I, Burke S, Berrada M, Cortes H. World Protests 20062013. Initiative for Policy Dialogue and Friedrich-EbertStiftung New York Working Paper 2013. (Accessed 201809-24). http://policydialogue.org/files/publications/papers/ World_Protests_2006-2013-Complete_and_Final_4282014. pdf

10. Rhainds M, Gaulin C, Goupil-Sormany I. Le Sommet des Amériques et la santé publique : plan de préparation et d'intervention - Résumé de présentation. BISE (Bulletin d'information en santé environnementale). juin 2002;13(3, supplément):3. http://collections.banq.qc.ca/ ark:/52327/bs26007

11. Kajita E, Luarca MZ, Wu H, Hwang B, Mascola L. Harnessing Syndromic Surveillance Emergency Department Data to Monitor Health Impacts During the 2015 Special Olympics World Games. Public Health Rep 2017 Jul/Aug;132(1_suppl Suppl 1):99S-105S. DOI PubMed

12. McCloskey B, Endericks $T$, Catchpole M, Zambon M, McLauchlin J, Shetty N, Manuel R, Turbitt D, Smith G, Crook $P$, Severi $E$, Jones J, Ibbotson S, Marshall R, Smallwood CA, Isla N, Memish ZA, Al-Rabeeah AA, Barbeschi M, Heymann DL, Zumla A. London 2012 Olympic and Paralympic Games: public health surveillance and epidemiology. Lancet 2014 Jun;383(9934):2083-9. DOI PubMed

13. CChan E, Hohenadel K, Lee B, Helferty M, Harris JR, Macdonald L, Badiani T. Surveillance de la santé publique pour les Jeux panaméricains et parapanaméricains de 2015 à Toronto. Relevé des maladies transmissibles au Canada 2017;43(7/8):175-84. DOI

14. Jorm LR, Thackway SV, Churches TR, Hills MW. Watching the Games: public health surveillance for the Sydney 2000 Olympic Games. J Epidemiol Community Health 2003 Feb;57(2):102-8. DOl PubMed

15. Tsouros AD, Efstathiou PA. Mass gatherings and public health: the experience of the Athens 2004 Olympic Games. World Health Organization Regional Office for Europe. WHO 2007 (Accédé 2018-09-24). http://www.euro.who.int/_data/ assets/pdf_file/0009/98415/E90712.pdf

16. Dapeng J, Ljungqvist A, Troedsson H. The Health Legacy of the 2008 Beijing Olympic Games: Successes and Recommendations. World Health Organization Regional Office for the Western Pacific. Geneva (CH): WHO 2010 (Accédé 2018-09-24). https://stillmed.olympic.org/ Documents/Commissions_PDFfiles/Medical_commission/ The_Health_Legacy_of_the_2008_Beijing_Olympic_Games. pdf

17. Takla A, Velasco E, Benzler J. The FIFA Women's World Cup in Germany 2011--a practical example for tailoring an event-specific enhanced infectious disease surveillance system. BMC Public Health 2012 Jul;12:576. DOI PubMed

18. Todkill D, Hughes HE, Elliot AJ, Morbey RA, Edeghere O, Harcourt S, Hughes T, Endericks T, McCloskey B, Catchpole M, Ibbotson S, Smith G. An Observational Study Using English Syndromic Surveillance Data Collected During the 2012 London Olympics - What did Syndromic Surveillance Show and What Can We Learn for Future Mass-gathering Events? Prehosp Disaster Med 2016 Dec;31(6):628-34. DOI PubMed 
19. Severi E, Heinsbroek E, Watson C, Catchpole M; HPA Olympics Surveillance Work Group. Infectious disease surveillance for the London 2012 Olympic and Paralympic Games. Euro Surveill 2012 Aug;17(31):20232. DOI PubMed

20. Alqahtani AS, BinDhim NF, Tashani M, Willaby HW, Wiley $K E$, Heywood AE, Booy R, Rashid H. Pilot use of a novel smartphone application to track traveller health behaviour and collect infectious disease data during a mass gathering: hajj pilgrimage 2014. J Epidemiol Glob Health 2016 Sep;6(3):147-55. DOl PubMed

21. Alotaibi BM, Yezli S, Bin Saeed AA, Turkestani A, Alawam $\mathrm{AH}$, Bieh KL. Strengthening health security at the Hajj mass gatherings: characteristics of the infectious diseases surveillance systems operational during the 2015 Hajj. J Travel Med 2017 May;24(3). DOI PubMed

22. Polkinghorne BG, Massey PD, Durrheim DN, Byrnes T, Maclntyre CR. Prevention and surveillance of public health risks during extended mass gatherings in rural areas: the experience of the Tamworth Country Music Festival, Australia. Public Health 2013 Jan;127(1):32-8. DOI PubMed

23. Hoy D, Saketa S, Maraka RR, Sio A, Wanyeki I, Frison P, Ogaoga D, Iniakawala D, Joshua C, Duituturaga S, Lepers C, Roth A, White P, Souares $Y$. Enhanced syndromic surveillance for mass gatherings in the Pacific: a case study of the 11th Festival of Pacific Arts in Solomon Islands, 2012. West Pac Surveill Response. 2016;7:15-20. https://read.qxmd.com/ journal/47699/3

24. Hick JL, Frascone RJ, Grimm K, Hillman M, Griffith J, Hogan M, Trotsky-Sirr R, Braun J. Health and medical preparedness and response to the 2008 Republican National Convention. Disaster Med Public Health Prep 2009 Dec;3(4):224-32. DOI PubMed

25. Sugishita $Y$, Ohkusa $Y$, Sugawara T, Shimatani N, Nadaoka Y, Kamiya N, Yasui Y, Taniguchi K, Okabe N. Enhanced Syndromic Surveillance for the Fourth Japan-China-South Korea Trilateral Summit 2011. J Bioterror Biodef 2013;4:1-7. $\mathrm{DOI}$

26. Agence de la santé publique du Canada. Bioterrorisme. Ottawa (ON): ASPC; 2004 (Accédé 2018-07-01). http://www. phac-aspc.gc.ca/ep-mu/aq-fra.php.php
27. Fleischauer AT, Gaines J. Enhancing Surveillance for Mass Gatherings: The Role of Syndromic Surveillance. Public Health Rep 2017 Jul/Aug;132(1_suppl Suppl 1):95S-8S. DOI PubMed

28. World Health Organization. Public health for mass gatherings: key considerations. Geneva (CH): WHO 2015. www.who.int/ihr/publications/WHO_HSE_GCR_2015.5/en/

29. Elliot AJ, Morbey RA, Hughes HE, Harcourt SE, Smith S, Loveridge P, Edeghere O, Ibbotson S, McCloskey B, Catchpole M, Smith GE. Syndromic surveillance - a public health legacy of the London 2012 Olympic and Paralympic Games. Public Health 2013 Aug;127(8):777-81. DOI PubMed

30. Berry AC. Syndromic surveillance and its utilisation for mass gatherings. Epidemiol Infect 2018 Jun;1-3:1-3. DOI PubMed

31. Thackway S, Churches T, Fizzell J, Muscatello D, Armstrong $P$. Should cities hosting mass gatherings invest in public health surveillance and planning? Reflections from a decade of mass gatherings in Sydney, Australia. BMC Public Health 2009 Sep;9:324. DOI PubMed

32. LégisQuébec. Loi sur la santé publique. 2001 (Accédé 2017-07-01). http://legisquebec.gouv.qc.ca/fr/ShowDoc/ $\mathrm{cs} / \mathrm{S}-2.2$

33. Lee LM, Steven M, Teutsch SM, Thacker SB, St-Louis ME. Principles and Practice of Public Health Surveillance. Oxford University Press 2010. DOI

34. Ministère de la Santé et des Services sociaux, Direction générale des services de santé et médecine universitaire. Cadre normatif pour le système d'information de gestion des urgences (SIGDU). 2009. http://numerique.banq.qc.ca/ patrimoine/details/52327/49642

35. Centers for Disease Control and Prevention. Surveillance Resource Center (Accédé 2019-01-21). www.cdc.gov/ surveillancepractice/index.html

36. Voxco Inc. Logiciel d'enquête flexible. www.voxco.com/fr/ 


\section{SCIENCE DE LA MISE EN OEUVRE}

\section{Annexe 1 : Raisons de consultation faisant l'objet d'une vigie dans les urgences, par catégorie}

\begin{tabular}{|c|c|}
\hline Catégorie & Raison de consultation \\
\hline \multirow[t]{2}{*}{ Cardiovasculaire } & Arrêt cardiaque (non traumatique) \\
\hline & Douleur thoracique angineuse \\
\hline \multirow[t]{4}{*}{ CBRNE/physique/environnemental } & Inhalation nocive \\
\hline & Exposition à un produit chimique \\
\hline & Intoxication \\
\hline & Exposition à une maladie contagieuse \\
\hline \multirow[t]{9}{*}{ Cutané/lymphatique } & Membre chaud et rouge \\
\hline & Edème/Douleur au cou \\
\hline & Douleur/Masse à l'aine \\
\hline & Prurit \\
\hline & Éruption cutanée \\
\hline & Edème ou rougeur localisée \\
\hline & Autre condition de la peau \\
\hline & Nodules, bosses, ampoules \\
\hline & Brûlure \\
\hline Fièvre & Fièvre \\
\hline \multirow[t]{6}{*}{ Gastro-intestinal } & Diarrhée \\
\hline & Nausées/Vomissements \\
\hline & Rectorragie/Méléna \\
\hline & Ictère/Jaunisse \\
\hline & Douleur abdominale \\
\hline & Diarrhée et fièvre \\
\hline \multirow[t]{4}{*}{ Hémorragique } & Épistaxis \\
\hline & Hématémèse \\
\hline & Hématurie \\
\hline & Ecchymose spontanée \\
\hline \multirow[t]{8}{*}{ Neurologique/musculaire } & Difficulté à avaler/Dysphagie \\
\hline & Altération du niveau de conscience \\
\hline & Confusion \\
\hline & Convulsion \\
\hline & Trouble d'équilibre/Ataxie \\
\hline & Faiblesse d'un membre/Symptômes d'accident vasculaire cérébral \\
\hline & Céphalée \\
\hline & Diplopie \\
\hline \multirow[t]{9}{*}{ Ophtalmologique/ Oto-rhino-laryngologique } & Douleur à la gorge \\
\hline & Exposition de l'œil à un produit chimique \\
\hline & Problème de vision \\
\hline & Écoulement/rougeur oculaire \\
\hline & Photophobie \\
\hline & Douleur oculaire \\
\hline & Traumatisme oculaire \\
\hline & Douleur oculaire \\
\hline & Traumatisme oculaire \\
\hline
\end{tabular}


Annexe 1 : Raisons de consultation faisant l'objet d'une vigie dans les urgences, par catégorie (suite)

\begin{tabular}{|l|l|}
\hline \multicolumn{5}{|c|}{ Catégorie } & \multicolumn{1}{c}{ Raison de consultation } \\
\hline \multirow{5}{*}{ Respiratoire } & Symptômes d'infection des voies respiratoires supérieures \\
\cline { 2 - 2 } & Douleur thoracique non angineuse \\
\hline & Dyspnée \\
\cline { 2 - 2 } & Arrêt respiratoire \\
\cline { 2 - 2 } & Toux/Congestion \\
\cline { 2 - 2 } & Hémoptysie \\
\hline \multirow{5}{*}{ Systémique/déshydratation } & Stridor \\
\hline & Sibilants-sans autre plainte \\
\hline & Toux et fièvre/Syndrome d'allure grippal \\
\hline & Faiblesse générale \\
\hline & Syncope/Pré-syncope \\
\hline & Oligo-anurie \\
\hline & Cyanose \\
\hline Abréviation:CBRNE, chimique, biologique, radiologique, nucléaire ou explosif
\end{tabular}


Annexe 2 : Diagnostics médicaux selon le code CIM-10 faisant l'objet d'une vigie dans les urgences par catégorie

\begin{tabular}{|c|c|c|c|}
\hline Catégorie & $\begin{array}{l}\text { Code } \\
\text { CIM-10 }\end{array}$ & Diagnostic CIM-10 & $\begin{array}{c}\text { Analyse } \\
\text { individuelle } \\
\text { effectuée (O/N) }\end{array}$ \\
\hline \multirow[t]{11}{*}{ Cardiovasculaire } & 120.0 & Angine instable & $\mathrm{N}$ \\
\hline & 120.9 & Angine de poitrine & $\mathrm{N}$ \\
\hline & 121.9 & Infarctus aigu du myocarde & $\mathrm{N}$ \\
\hline & 124.9 & Cardiopathie ischémique aigue, Syndrome coronarien aigu (SCA) & $\mathrm{N}$ \\
\hline & 146.9 & Arrêt cardiaque & $\mathrm{N}$ \\
\hline & 195.9 & Hypotension & $\mathrm{N}$ \\
\hline & R00.1 & Bradycardie & $\mathrm{N}$ \\
\hline & $\mathrm{R} 07.2$ & Douleur thoracique d'étiologie indéterminée (DTEI) & $\mathrm{N}$ \\
\hline & R07.3 & Douleur rétro-sternale (DRS) & $\mathrm{N}$ \\
\hline & R07.4 & Douleur thoracique & $\mathrm{N}$ \\
\hline & R57.0 & Choc cardiogénique & $\mathrm{N}$ \\
\hline \multirow{17}{*}{$\begin{array}{l}\text { CBRNE/physique/ } \\
\text { environnemental }\end{array}$} & J68.9 & Inhalation de produits toxiques & 0 \\
\hline & T59.9 & Effet toxique des fumées/gaz & O \\
\hline & T62.9 & Effet toxique alimentaire & O \\
\hline & T67.0 & Coup de chaleur et insolation & O \\
\hline & T52.9 & Effet toxique d'un solvant organique & $\mathrm{N}$ \\
\hline & T54.9 & Effet toxique d'une substance corrosive & $\mathrm{N}$ \\
\hline & T58 & Effet toxique du monoxyde de carbone & $\mathrm{N}$ \\
\hline & T60.9 & Effet toxique d'un pesticide & $\mathrm{N}$ \\
\hline & T65.9 & Effet toxique d'une substance & $\mathrm{N}$ \\
\hline & T66 & Effets de radiation & $\mathrm{N}$ \\
\hline & T67.9 & Effet de la chaleur et de lumière & $\mathrm{N}$ \\
\hline & $\mathrm{T71}$ & Asphyxie & $\mathrm{N}$ \\
\hline & Y14 & $\begin{array}{l}\text { Intoxication par des médicaments et substances biologiques, autres et sans } \\
\text { précision et exposition à ces produits, intention non déterminée }\end{array}$ & $\mathrm{N}$ \\
\hline & Y17 & $\begin{array}{l}\text { Intoxication par d'autres gaz et émanations et exposition à ces produits, } \\
\text { intention non déterminée }\end{array}$ & $\mathrm{N}$ \\
\hline & Y25 & Contact avec matériel explosif, intention non déterminée & $\mathrm{N}$ \\
\hline & Z20.9 & Exposition à une maladie transmissible & $\mathrm{N}$ \\
\hline & Z29.9 & Mesure prophylactique & $\mathrm{N}$ \\
\hline
\end{tabular}


Annexe 2 : Diagnostics médicaux selon le code CIM-10 faisant l'objet d'une vigie dans les urgences par catégorie (suite)

\begin{tabular}{|c|c|c|c|}
\hline Catégorie & $\begin{array}{l}\text { Code } \\
\text { CIM-10 }\end{array}$ & Diagnostic CIM-10 & $\begin{array}{c}\text { Analyse } \\
\text { individuelle } \\
\text { effectuée }(\mathrm{O} / \mathrm{N})\end{array}$ \\
\hline \multirow[t]{23}{*}{ Cutané/lymphatique } & B05.9 & Rougeole & 0 \\
\hline & B09 & Exanthème viral & O \\
\hline & L02.9 & Abcès/furoncle/anthrax & 0 \\
\hline & 188.9 & Lymphadénite non spécifique & $\mathrm{N}$ \\
\hline & 189.1 & Lymphangite & $\mathrm{N}$ \\
\hline & L03.9 & Cellulite & $\mathrm{N}$ \\
\hline & L13.9 & Maladie bulleuse & $\mathrm{N}$ \\
\hline & L25.9 & Unspecified contact dermatitis & $\mathrm{N}$ \\
\hline & L29.9 & Pruritus & $\mathrm{N}$ \\
\hline & L50.9 & Urticaria & $\mathrm{N}$ \\
\hline & L51.9 & Erythema multiforme & $\mathrm{N}$ \\
\hline & L72.9 & Cyst of skin & $\mathrm{N}$ \\
\hline & L97 & Ulcer of lower limb, diabetic foot ulcer & $\mathrm{N}$ \\
\hline & L98.9 & Disorder of skin and subcutaneous tissue & $\mathrm{N}$ \\
\hline & M72.69 & Necrotizing fasciitis & $\mathrm{N}$ \\
\hline & $\mathrm{R} 21$ & Éruption cutanée & $\mathrm{N}$ \\
\hline & R22.9 & Tuméfaction/masse localisée & $\mathrm{N}$ \\
\hline & R59.9 & Adénopathie & $\mathrm{N}$ \\
\hline & R60.0 & Edème localisé & $\mathrm{N}$ \\
\hline & $\mathrm{T} 29.0$ & Brûlures multiples & $\mathrm{N}$ \\
\hline & T30.1 & Brûlure du premier degré & $\mathrm{N}$ \\
\hline & T30.2 & Brûlure du second degré & $\mathrm{N}$ \\
\hline & T30.3 & Brûlure du troisième degré & $\mathrm{N}$ \\
\hline \multirow[t]{14}{*}{ Gastro-intestinal } & A05.9 & Intoxication alimentaire bactérienne & 0 \\
\hline & A09.9 & Gastroentérite & O \\
\hline & B19.9 & Hépatite virale & O \\
\hline & K29.7 & Gastrite & $\mathrm{N}$ \\
\hline & K29.9 & Gastroduodénite & $\mathrm{N}$ \\
\hline & K51.9 & Rectocolite hémorragique & $\mathrm{N}$ \\
\hline & K56.7 & lléus & $\mathrm{N}$ \\
\hline & K65.0 & Péritonite aiguë & $\mathrm{N}$ \\
\hline & K72.9 & Insuffisance hépatique & $\mathrm{N}$ \\
\hline & K92.9 & Maladie du système digestif & $\mathrm{N}$ \\
\hline & R10.4 & Douleur abdominale & $\mathrm{N}$ \\
\hline & R11.1 & Nausées isolées & $\mathrm{N}$ \\
\hline & $\mathrm{R} 11.3$ & Nausées avec vomissements & $\mathrm{N}$ \\
\hline & $\mathrm{R} 17$ & Ictère & $\mathrm{N}$ \\
\hline
\end{tabular}


Annexe 2 : Diagnostics médicaux selon le code CIM-10 faisant l'objet d'une vigie dans les urgences par catégorie (suite)

\begin{tabular}{|c|c|c|c|}
\hline Catégorie & $\begin{array}{l}\text { Code } \\
\text { CIM-10 }\end{array}$ & Diagnostic CIM-10 & $\begin{array}{c}\text { Analyse } \\
\text { individuelle } \\
\text { effectuée }(\mathrm{O} / \mathrm{N})\end{array}$ \\
\hline \multirow[t]{10}{*}{ Hémorragique } & D65 & CIVD-Coagulation intravasculaire disséminée & $\mathrm{N}$ \\
\hline & D68.9 & Anomalie de la coagulation & $\mathrm{N}$ \\
\hline & D69.6 & Thrombopénie & $\mathrm{N}$ \\
\hline & D75.9 & Hémopathie & $\mathrm{N}$ \\
\hline & 162.9 & Hémorragie intracranienne sans trauma & $\mathrm{N}$ \\
\hline & K92.0 & Hématémèse & $\mathrm{N}$ \\
\hline & K92.2 & Hémorragie digestive & $\mathrm{N}$ \\
\hline & R04.0 & Épistaxis & $\mathrm{N}$ \\
\hline & R31.8 & Hématurie & $\mathrm{N}$ \\
\hline & R58 & Hémorragie & $\mathrm{N}$ \\
\hline \multirow[t]{28}{*}{ Infectieux } & A39.2 & Méningococcémie aiguë & 0 \\
\hline & A21.2 & Tularémie pulmonaire & O \\
\hline & A00.0 & A Vibrio cholerae 01, biovar cholerae & $\mathrm{N}$ \\
\hline & A00.1 & A Vibrio cholerae 01, biovar El Tor & $\mathrm{N}$ \\
\hline & A00.9 & Choléra, sans précision & $\mathrm{N}$ \\
\hline & A01.0 & Fièvre typhoïde & $\mathrm{N}$ \\
\hline & A01.1 & Paratyphoïde A & $\mathrm{N}$ \\
\hline & A01.2 & Paratyphoïde B & $\mathrm{N}$ \\
\hline & A01.3 & Paratyphoïde C & $\mathrm{N}$ \\
\hline & A01.4 & Paratyphoïde, sans précision & $\mathrm{N}$ \\
\hline & A02.0 & Entérite à Salmonella & $\mathrm{N}$ \\
\hline & A02.1 & Septicémie à Salmonella & $\mathrm{N}$ \\
\hline & A02.2 & Infection localisée à Salmonella & $\mathrm{N}$ \\
\hline & A02.8 & Autres infections précisées à Salmonella & $\mathrm{N}$ \\
\hline & A02.9 & Salmonellose, sans précision & $\mathrm{N}$ \\
\hline & A03.0 & Shigellose à Shigella dysenteriae & $\mathrm{N}$ \\
\hline & A03.1 & Shigellose à Shigella flexneri & $\mathrm{N}$ \\
\hline & A03.2 & Shigellose à Shigella boydii & $\mathrm{N}$ \\
\hline & A03.3 & Shigellose à Shigella sonnei & $\mathrm{N}$ \\
\hline & A03.8 & Autres shigelloses & $\mathrm{N}$ \\
\hline & A03.9 & Shigellose, sans précision & $\mathrm{N}$ \\
\hline & A15.0 & $\begin{array}{l}\text { Tuberculose pulmonaire, confirmée par examen microscopique de } \\
\text { l'expectoration, avec ou sans culture }\end{array}$ & $\mathrm{N}$ \\
\hline & A15.1 & Tuberculose pulmonaire, confirmée par culture seulement & $\mathrm{N}$ \\
\hline & A15.2 & Tuberculose pulmonaire, avec confirmation histologique & $\mathrm{N}$ \\
\hline & A15.3 & Tuberculose pulmonaire, avec confirmation, moyen non précisé & $\mathrm{N}$ \\
\hline & A15.4 & $\begin{array}{l}\text { Tuberculose des ganglions intrathoraciques, avec confirmation bactériologique } \\
\text { et histologique }\end{array}$ & $\mathrm{N}$ \\
\hline & A15.5 & $\begin{array}{l}\text { Tuberculose du larynx, de la trachée et des bronches, avec confirmation } \\
\text { bactériologique et histologique }\end{array}$ & $\mathrm{N}$ \\
\hline & A15.6 & Pleurésie tuberculeuse, avec confirmation bactériologique et histologique & $\mathrm{N}$ \\
\hline
\end{tabular}


Annexe 2 : Diagnostics médicaux selon le code CIM-10 faisant l'objet d'une vigie dans les urgences par catégorie (suite)

\begin{tabular}{|c|c|c|c|}
\hline Catégorie & $\begin{array}{l}\text { Code } \\
\text { CIM-10 }\end{array}$ & Diagnostic CIM-10 & $\begin{array}{c}\text { Analyse } \\
\text { individuelle } \\
\text { effectuée }(\mathrm{O} / \mathrm{N})\end{array}$ \\
\hline \multirow[t]{28}{*}{ Infectieux (suite) } & A15.7 & $\begin{array}{l}\text { Primo-infection tuberculeuse de l'appareil respiratoire, avec confirmation } \\
\text { bactériologique et histologique }\end{array}$ & $\mathrm{N}$ \\
\hline & A15.8 & $\begin{array}{l}\text { Autres formes de tuberculose de l'appareil respiratoire, avec confirmation } \\
\text { bactériologique et histologique }\end{array}$ & $\mathrm{N}$ \\
\hline & A15.9 & $\begin{array}{l}\text { Tuberculose de l'appareil respiratoire sans précision, avec confirmation } \\
\text { bactériologique et histologique }\end{array}$ & $\mathrm{N}$ \\
\hline & A20.2 & Peste pulmonaire & $\mathrm{N}$ \\
\hline & A36.0 & Diphtérie pharyngée & $\mathrm{N}$ \\
\hline & A36.1 & Diphtérie rhinopharyngée & $\mathrm{N}$ \\
\hline & A36.2 & Diphtérie laryngée & $\mathrm{N}$ \\
\hline & A36.3 & Diphtérie cutanée & $\mathrm{N}$ \\
\hline & A36.8 & Autres formes de diphtérie & $\mathrm{N}$ \\
\hline & A36.9 & Diphtérie, sans précision & $\mathrm{N}$ \\
\hline & A40.9 & Infection à streptocoque & $\mathrm{N}$ \\
\hline & A41.9 & Septicémie & $\mathrm{N}$ \\
\hline & A48.3 & Choc toxique & $\mathrm{N}$ \\
\hline & A49.9 & Bactériémie & $\mathrm{N}$ \\
\hline & A80.0 & Poliomyélite paralytique aiguë, associée au virus vaccinal & $\mathrm{N}$ \\
\hline & A80.1 & Poliomyélite paralytique aiguë, virus sauvage importé & $\mathrm{N}$ \\
\hline & A80.2 & Poliomyélite paralytique aiguë, virus sauvage indigène & $\mathrm{N}$ \\
\hline & A80.3 & Poliomyélites paralytiques aiguës, autres et sans précision & $\mathrm{N}$ \\
\hline & A80.4 & Poliomyélite aiguë non paralytique & $\mathrm{N}$ \\
\hline & A80.9 & Poliomyélite aiguë, sans précision & $\mathrm{N}$ \\
\hline & A96.2 & Fièvre de Lassa & $\mathrm{N}$ \\
\hline & A98.0 & Fièvre hémorragique de Crimée [du Congo] & $\mathrm{N}$ \\
\hline & A98.3 & Maladie de Marburg & $\mathrm{N}$ \\
\hline & A98.4 & Maladie à virus Ébola & $\mathrm{N}$ \\
\hline & B03 & Variole & $\mathrm{N}$ \\
\hline & B34.9 & Infection virale & $\mathrm{N}$ \\
\hline & R50.9 & Fièvre & $\mathrm{N}$ \\
\hline & R57.2 & Choc septique & $\mathrm{N}$ \\
\hline \multirow[t]{3}{*}{ Intoxication opioïdes } & $\mathrm{T} 40.1$ & Intoxication à l'héroïne & $\mathrm{N}$ \\
\hline & $\mathrm{T} 40.6$ & Intoxication aux narcotiques & $\mathrm{N}$ \\
\hline & F11.9 & Troubles mentaux liés aux opiacés & $\mathrm{N}$ \\
\hline
\end{tabular}


Annexe 2 : Diagnostics médicaux selon le code CIM-10 faisant l'objet d'une vigie dans les urgences par catégorie (suite)

\begin{tabular}{|c|c|c|c|}
\hline Catégorie & $\begin{array}{c}\text { Code } \\
\text { CIM-10 }\end{array}$ & Diagnostic CIM-10 & $\begin{array}{c}\text { Analyse } \\
\text { individuelle } \\
\text { effectuée }(\mathrm{O} / \mathrm{N})\end{array}$ \\
\hline \multirow[t]{31}{*}{ Neurologique/musculaire } & A05.1 & Botulisme & O \\
\hline & A39.0 & Méningite à méningocoque & 0 \\
\hline & A86 & Encéphalite virale & $\mathrm{N}$ \\
\hline & A87.9 & Méningite virale & $\mathrm{N}$ \\
\hline & F05.9 & Delirium & $\mathrm{N}$ \\
\hline & G00.9 & Méningite bactérienne & $\mathrm{N}$ \\
\hline & G03.9 & Méningite autre & $\mathrm{N}$ \\
\hline & G04.9 & Encéphalomyélite & $\mathrm{N}$ \\
\hline & G24.9 & Dystonie & $\mathrm{N}$ \\
\hline & G41.9 & Status épilepticus & $\mathrm{N}$ \\
\hline & G44.8 & Céphalée, autre & $\mathrm{N}$ \\
\hline & G51.0 & Paralysie de Bell & $\mathrm{N}$ \\
\hline & G52.9 & Affection des nerfs crâniens & $\mathrm{N}$ \\
\hline & G61.0 & Syndrome de Guillain-Barré & $\mathrm{N}$ \\
\hline & G62.9 & Polynévrite & $\mathrm{N}$ \\
\hline & G72.9 & Myopathie & $\mathrm{N}$ \\
\hline & G83.4 & Syndrome de la queue de cheval & $\mathrm{N}$ \\
\hline & G83.9 & Paralysie & $\mathrm{N}$ \\
\hline & G93.4 & Encéphalopathie & $\mathrm{N}$ \\
\hline & G95.9 & Myélopathie & $\mathrm{N}$ \\
\hline & G96.9 & Affection du système nerveux central, autre & $\mathrm{N}$ \\
\hline & H53.2 & Diplopie & $\mathrm{N}$ \\
\hline & M62.99 & Myopathie & $\mathrm{N}$ \\
\hline & R13.8 & Dysphagie & $\mathrm{N}$ \\
\hline & R26.88 & Anomalie de la démarche/mobilité & $\mathrm{N}$ \\
\hline & $\mathrm{R} 29.8$ & Symptômes relatifs aux systèmes nerveux et ostéo-musculaire & $\mathrm{N}$ \\
\hline & R40.0 & Atteinte de l'état de conscience & $\mathrm{N}$ \\
\hline & R40.29 & Coma & $\mathrm{N}$ \\
\hline & R41.0 & Désorientation & $\mathrm{N}$ \\
\hline & R51 & Céphalée & $\mathrm{N}$ \\
\hline & R56.88 & Convulsions & $\mathrm{N}$ \\
\hline
\end{tabular}


Annexe 2 : Diagnostics médicaux selon le code CIM-10 faisant l'objet d'une vigie dans les urgences par catégorie (suite)

\begin{tabular}{|c|c|c|c|}
\hline Catégorie & $\begin{array}{l}\text { Code } \\
\text { CIM-10 }\end{array}$ & Diagnostic CIM-10 & $\begin{array}{c}\text { Analyse } \\
\text { individuelle } \\
\text { effectuée }(\mathrm{O} / \mathrm{N})\end{array}$ \\
\hline \multirow{14}{*}{$\begin{array}{l}\text { Ophtalmologique/ } \\
\text { Oto-rhino-aryngologique }\end{array}$} & H16.0 & Ulcère cornéen & $\mathrm{N}$ \\
\hline & $\mathrm{H} 10.9$ & Conjonctivite & $\mathrm{N}$ \\
\hline & $\mathrm{H} 16.9$ & Kératite, non précisé & $\mathrm{N}$ \\
\hline & H18.9 & Affection cornéenne & $\mathrm{N}$ \\
\hline & H53.9 & Trouble de la vision & $\mathrm{N}$ \\
\hline & $\mathrm{H} 57.1$ & Douleur oculaire & $\mathrm{N}$ \\
\hline & H57.9 & Affection œil et annexes & $\mathrm{N}$ \\
\hline & J02.9 & Pharyngite aiguë & $\mathrm{N}$ \\
\hline & J03.9 & Amygdalite aiguë & $\mathrm{N}$ \\
\hline & J04.0 & Laryngite aiguë & $\mathrm{N}$ \\
\hline & J05.0 & Croup-Laryngite obstructive aiguë & $\mathrm{N}$ \\
\hline & J05.1 & Épiglottite aiguë & $\mathrm{N}$ \\
\hline & R07.0 & Mal de gorge & $\mathrm{N}$ \\
\hline & S05.9 & Trauma de l'œil/orbite & $\mathrm{N}$ \\
\hline \multirow[t]{18}{*}{ Respiratoire } & J06.9 & IVRS-Infection des voies respiratoires supérieures & O \\
\hline & U04.90 & SRAS-Syndrome respiratoire aigu sévère suspecté & 0 \\
\hline & J04.1 & Trachéite aiguë & $\mathrm{N}$ \\
\hline & $\mathrm{J} 04.2$ & Laryngotrachéite aiguë & $\mathrm{N}$ \\
\hline & $\mathrm{J} 11.8$ & Grippe/influenza & $\mathrm{N}$ \\
\hline & $\mathrm{J} 18.9$ & Pneumonie & $\mathrm{N}$ \\
\hline & $\mathrm{J} 20.9$ & Bronchite aiguë & $\mathrm{N}$ \\
\hline & $\mathrm{J} 45.90$ & Asthme & $\mathrm{N}$ \\
\hline & $\mathrm{J} 69.0$ & Pneumopathie d'inhalation & $\mathrm{N}$ \\
\hline & $\mathrm{J} 80$ & SDRA-Syndrome de détresse respiratoire aiguë & $\mathrm{N}$ \\
\hline & J96.0 & Insuffisance respiratoire aiguë & $\mathrm{N}$ \\
\hline & J98.0 & Bronchospasme & $\mathrm{N}$ \\
\hline & J98.9 & Troubles respiratoires, autres & $\mathrm{N}$ \\
\hline & $\mathrm{R} 04.2$ & Hémoptysie & $\mathrm{N}$ \\
\hline & R05 & Toux & $\mathrm{N}$ \\
\hline & R06.0 & Dyspnée & $\mathrm{N}$ \\
\hline & R06.1 & Stridor & $\mathrm{N}$ \\
\hline & R09.2 & Arrêt respiratoire & $\mathrm{N}$ \\
\hline \multirow{11}{*}{$\begin{array}{l}\text { Systémique/ } \\
\text { déshydratation }\end{array}$} & E86.0 & Déshydratation & $\mathrm{N}$ \\
\hline & E87.0 & Hypernatrémie & $\mathrm{N}$ \\
\hline & E87.1 & Hyponatrémie & $\mathrm{N}$ \\
\hline & E87.6 & Hypokaliémie & $\mathrm{N}$ \\
\hline & E87.8 & Déséquilibres hydro-électrolytiques & $\mathrm{N}$ \\
\hline & N17.9 & IRA-Insuffisance rénale aiguë & $\mathrm{N}$ \\
\hline & $\mathrm{R} 23.0$ & Cyanose & $\mathrm{N}$ \\
\hline & R53 & Malaise et fatigue & $\mathrm{N}$ \\
\hline & R55 & Syncope/Lipothymie & $\mathrm{N}$ \\
\hline & R57.1 & Choc hypovolémique & $\mathrm{N}$ \\
\hline & R57.9 & Choc & $\mathrm{N}$ \\
\hline
\end{tabular}

